PROCEEDINGS OF THE

AMERICAN MATHEMATICAL SOCIETY

Volume 134, Number 7, Pages 1899-1908

S 0002-9939(06)08215-3

Article electronically published on January 17, 2006

\title{
THE GROUPOID ALGEBRA OF AN EIGENVALUE PATTERN
}

\author{
KIN-WAI TSANG
}

(Communicated by David R. Larson)

\begin{abstract}
The eigenvalue pattern of a *-homomorphism between two matrix algebras over commutative $\mathrm{C}^{*}$-algebras is a generalization of the Gelfand map in the commutative case. We give a systematic formulation of abstract eigenvalue pattern and extend the classical results by using a technique involving the groupoid algebras of eigenvalue patterns. In the case with matrix algebras over the one-dimensional circle, we characterize all the *-homomorphisms up to unitary equivalence by their eigenvalue patterns. Moreover, this technique has an application to recent classification theorems of $\mathrm{C}^{*}$-algebras proved by the present author.
\end{abstract}

\section{INTRODUCTION}

Let there be given two compact Hausdorff spaces $X$ and $Y$. The so-called Gelfand map characterizes all the homomorphisms between the $\mathrm{C}^{*}$-algebra of continuous complex-valued functions on $X, C(X)$, and that on $Y, C(Y)$, by continuous maps between $Y$ and $X$. More precisely, every continuous map $\phi$ from $Y$ to $X$ induces a canonical map $\phi^{*}$ from $C(X)$ to $C(Y)$, with $\phi^{*}(f)(y)$ equal to $f(\phi(y))$ for all $f$ in $C(X)$ and $y$ in $Y$. We shall show that homomorphisms between matrix algebras over $C(X)$ and $C(Y)$ can be systemized in an analogous manner.

For any homomorphism $\phi$ from $C(X)$ to $M_{k}(C(Y))$, the $k$-dimensional matrix algebra over $C(Y), \phi$ induces a canonical multi-valued map $\phi^{*}$ from $Y$, the spectrum of $M_{k}(C(Y))$, to $X$, the spectrum of $C(X)$ : for any $y$ in $Y,(\phi(\cdot))(y): C(X) \rightarrow$ $M_{k}(\mathbb{C})$ is a $k$-dimensional representation of $C(X)$. So it is a direct sum of irreducible representations of $C(X)$. Now, since all irreducible representations of $C(X)$ are evaluations at some elements of $X$, we can associate $k$ elements $x_{1}, \ldots, x_{k}$ of $X$ (which may be repeated) to the representation $\phi(\cdot)(y)$. The map $y \mapsto\left\{x_{1}, \ldots, x_{k}\right\}$ is called the eigenvalue pattern of $\phi$ and is denoted by $\phi^{*}$. A question arises:

(Q1) Can any multi-valued map (continuous in some sense) be the eigenvalue pattern of some homomorphism?

In the case $k=1$, the Gelfand map gives an affirmative answer to the above question.

The present paper is the first paper giving the definition of a singular abstract eigenvalue pattern (a similar concept in the full matrix algebra (non-singular) case is considered in $\mathrm{GO}$ ). We will give partial answers to the above question in the

Received by the editors June 1, 2003 and, in revised form, February 1, 2005.

2000 Mathematics Subject Classification. Primary 46L05; Secondary 46L35.

Key words and phrases. Groupoid algebra, path space, singular eigenvalue pattern, Gelfand map.

(C)2006 American Mathematical Society Reverts to public domain 28 years from publication 
higher-dimensional case by using a technique involving the groupoid algebras of eigenvalue patterns. In particular, in the case with matrix algebras over the onedimensional circle, we give a complete answer to the above question (Corollary 7.1). In other words, we characterize all the *-homomorphisms up to unitary equivalence by their eigenvalue patterns.

This technique has an application to recent classification theorems of $\mathrm{C}^{*}$-algebras proved by the present author (cf. Ts1, Ts2]). In Ts2, the present author applies Theorem 9.1 to construct a family of *-homomorphisms. This family of $*_{-}$ homomorphisms turns out to be a useful technique for proving the range of the invariant theorem in Ts2], which is the first Effros-Handelman-Shen-type theorem (cf. EHS) associated with an isomorphism theorem for stably projectionless $\mathrm{C}^{*}$ algebras. (In [Ell2] and [EV], there are similar results, but the constructions of these authors rely on the complicated structure of the fibre at infinity of their building block algebra. In [s2], the fibre at the infinity of the building block algebra (cf. Chapter 8 in the present paper) is simple (its spectrum has only a single point) and the construction in [Ts2] is more natural, and also perhaps easier to understand).

The convenience of using the eigenvalue pattern can be seen in Theorem 9.1, which can be treated (with some technical modifications) as a new and shorter proof of Razak's existence theorem ( $[\mathrm{Ra}$, Theorem 3.1). We can freely deduce the complicated work on *-homomorphism construction by considering only the eigenvalue pattern construction. This gives us more flexibility in doing approximate intertwining arguments in classification theorem (cf. Ell1]).

\section{NON-SINGULAR ABSTRACT EIGENVALUE PATTERN}

Let $X$ and $Y$ be compact Hausdorff topological spaces. We denote the family of finite subsets (with multiplicity; that is, some elements can be repeated) of $X$ with $k$ elements by $P^{k}(X)$. Then $P^{k}(X)$ has a canonical topology, namely the Gromov-Hausdorff topology (that is, the product topology modulo the permutation relation).

A $k$-dimensional non-singular abstract eigenvalue pattern from $Y$ to $X$ is a continuous map $\psi$ from $Y$ to $P^{k}(X)$.

Remarks.

(1) Non-singular means the dimension $k$ of the image of $\psi$ is fixed for all $y$ in $Y$. In Section 9, we shall discuss a singular case.

(2) If $\phi^{*}$ is the eigenvalue pattern of a homomorphism $\phi: C(X) \rightarrow M_{k}(C(Y))$, then by continuity of $\phi$, the eigenvalue pattern $\phi^{*}$ is clearly a non-singular abstract eigenvalue pattern.

We shall introduce a technique involving the groupoid algebras of eigenvalue patterns.

\section{Groupoid ALGeBra}

We review some definitions of a groupoid and its algebra in this section; more details can be found in $\mathrm{Re}$.

A groupoid $G$ is a set with:

(1) a product map $(x, y) \mapsto x y: G^{2} \rightarrow G$, where $G^{2}(\subset G \times G)$ is the set all composable pairs,

(2) an inverse map $x \mapsto x^{-1}: G \rightarrow G$, 
satisfying:

i) $\left(x^{-1}\right)^{-1}=x$,

ii) $(x, y)$ and $(y, z) \in G^{2} \Rightarrow(x y, z)$ and $(x, y z) \in G^{2}$ and $(x y) z=x(y z)$,

iii) $\left(x^{-1}, x\right) \in G^{2}$ and if $(x, y) \in G^{2}$, then $x^{-1}(x y)=y,\left(x, x^{-1}\right) \in G^{2}$ and if $(z, x) \in G^{2}$, then $(z x) x^{-1}=z$.

We call an element $x$ in $G$ an arrow, and, for $x \in G, h(x)=x x^{-1}$ is the head of $x$ and $t(x)=x^{-1} x$ is the tail of $x$. The set $G^{0}=h(G)=t(G)$ is called the unit space (or the set of objects) of $G$.

A topological groupoid $G$ is a groupoid as well as a topological space such that the inverse map and the product map are continuous when $G^{2}$ has the induced topology from $G \times G$.

A locally compact groupoid $G$ is called étale if its head and tail maps are local homeomorphisms from $G$ to $G^{0}$.

Let $G$ be a locally compact groupoid. For $f, g$ in $C_{c}(G)$ (continuous function on $G$ with compact support), let us define the convolution of $f$ and $g$, and the involution of $f$ as $f * g(x)=\sum_{y \in G} f(x y) g\left(y^{-1}\right)$ and $f^{*}(x)=\overline{f\left(x^{-1}\right)}$, respectively. Then $C_{c}(G)$ becomes a topological *-algebra (whenever $C_{c}(G)$ is closed under convolution) - in particular, it is true when the groupoid is étale, see ([Re], Proposition 1.1).

Suppose that $C_{c}(G)$ is closed under convolution. A representation of $C_{c}(G)$ on a Hilbert space $H$ is a (continuous) *-homomorphism $L: C_{c}(G) \rightarrow B(H)$ (bounded operators over $H$ ), when $C_{c}(G)$ has the inductive limit topology and $B(H)$ the weak operator topology, such that the linear span of $\left\{L(f) h: f \in C_{c}(G), h \in H\right\}$ is dense in $H$. A representation $L$ of $C_{c}(G)$ is called bounded if $\|L(f)\|_{H} \leq\|f\|_{C_{c}(G)}$ for all $f$ in $C_{c}(G)$.

If we define the norm $\|f\|$ equal to $\sup _{L}$ bounded $\|L(f)\|$, then the norm $\|\cdot\|$ is a $\mathrm{C}^{*}$-norm ( $\left[\mathrm{Re}\right.$, Proposition 1.10). We denote the completion of $C_{c}(G)$ with respect to the above $\mathrm{C}^{*}$-norm as $C^{*}(G)$.

\section{The PATH SPACE OF A NON-Singular ABSTRACT EIGENVAlue PATtern}

The idea of path space was considered by others many years ago (cf. Eva]); typical non-trivial examples are path spaces of the Bratteli diagrams associated with approximate finite algebras, AF-algebras (cf. $\mathrm{Br}$ ).

For a given non-singular abstract eigenvalue pattern $\psi: Y \rightarrow P^{k}(X)$. We define the path space to be the set

$$
E^{\psi}=\left\{(s, r): r \in Y, s \in\left\{x_{1}, \ldots, x_{k}\right\},\left[x_{1}, \ldots, x_{k}\right]=\psi(r)\right\} .
$$

It is a set of pairs with multiplicity (some elements are repeated if $s$ appears in the image of $\psi$ more than once). For any element $e=(s, r)$ in $E^{\psi}$, we write $r(e)=r$ (the range of $e$ ) and $s(e)=s$ (the source of $e$ ). An equivalent relation $\sim$ can be defined on $E^{\psi}$ by $e_{1} \sim e_{2}$ if $r\left(e_{1}\right)=r\left(e_{2}\right)$ for $e_{1}, e_{2}$ in $E^{\psi}$ (two paths are related if and only if they go to the same element in $Y)$.

\section{The GROUPOID OF A PATH SPACE}

Let $\psi, E^{\psi}$ be the same as in the previous sections. The equivalent relation $\sim$ on $E^{\psi}$ induces a groupoid $G_{E^{\psi}}$. More precisely, we write an element in $G_{E^{\psi}}$ as $\left(e_{1}, e_{2}\right)$ (an element of the graph of the relation $\sim$ ), and we define the inverse operation as $\left(e_{1}, e_{2}\right)^{-1}=\left(e_{2}, e_{1}\right)$ and the product operation as $\left(e_{1}, e_{2}\right)\left(e_{2}, e_{3}\right)=\left(e_{1}, e_{3}\right)$. Two pairs $\left(e_{1}, e_{2}\right)$ and $\left(e_{1}^{\prime}, e_{2}^{\prime}\right)$ are composable if and only if $e_{2}$ equals $e_{1}^{\prime}$. 


\section{Global Labelling Property}

Let there be given a non-singular abstract eigenvalue pattern $\psi: Y \rightarrow P^{k}(X)$ (with $X, Y$ being compact and Hausdorff). The map $\psi$ satisfies the Global Labelling Property if there exist continuous maps $\psi_{1}, \ldots, \psi_{k}$ from $Y$ to $X$, such that $\psi(y)=$ $\left\{\psi_{1}(y), \ldots, \psi_{k}(y)\right\}$ up to permutation. Clearly, a labelling may not be unique.

For an abstract eigenvalue pattern $\psi$ satisfying the global labelling property with labelling $\left(\psi_{1}, \ldots, \psi_{k}\right)$, we can distinguish the elements in $E^{\psi}$ (and hence the elements in $\left.G_{E^{\psi}}\right)$. More precisely, for any $y$ in $Y$, suppose that $\psi_{i}(y)$ equals $\psi_{j}(y)$ as an element in $X$ with $i \neq j$. Now with the help of the labelling, we can regard the elements $\left(\Psi_{i}(y), y\right)$ and $\left(\psi_{j}(y), y\right)$ as different elements in $E^{\psi}$; hence, the elements $\left(\left(\psi_{i}(y), y\right),\left(\psi_{i}(y), y\right)\right),\left(\left(\psi_{i}(y), y\right),\left(\psi_{j}(y), y\right)\right),\left(\left(\psi_{j}(y), y\right),\left(\psi_{i}(y), y\right)\right)$ and $\left(\left(\psi_{j}(y), y\right),\left(\psi_{j}(y), y\right)\right)$ are four different elements in $G_{E^{\psi}}$.

Furthermore, we can define a topology on $G_{E^{\psi}}$ using a fixed labelling $\left(\psi_{1}, \ldots, \psi_{k}\right)$ of the eigenvalue pattern $\psi$ : for an element $\left(e_{1}, e_{2}\right)$ in $G_{E^{\psi}}$ with $s\left(e_{1}\right)=\psi_{i_{1}}\left(r\left(e_{1}\right)\right)$ and $s\left(e_{2}\right)=\psi_{i_{2}}\left(r\left(e_{2}\right)\right)$, and an open neighbourhood $U$ of $r\left(e_{1}\right)=r\left(e_{2}\right)$ in $Y$, we define

$$
O\left(\left(e_{1}, e_{2}\right), U\right):=\left\{\left(e, e^{\prime}\right): r(e)=r\left(e^{\prime}\right) \in U, s(e) \in \psi_{i_{1}}^{-1}(U), s\left(e^{\prime}\right) \in \psi_{i_{2}}^{-1}(U)\right\} .
$$

Topology on the groupoid $G_{E^{\psi}}$ is generated by such sets (the sets $O\left(\left(e_{1}, e_{2}\right), U\right)$ 's are basic neighbourhoods of $\left.\left(e_{1}, e_{2}\right)\right)$.

Proposition 6.1. Let $X$ and $Y$ be compact Hausdorff topological spaces and let $\psi: Y \rightarrow P^{k}(X)$ be a non-singular abstract eigenvalue pattern satisfying the global labelling property. The groupoid $G_{E^{\psi}}$, associated with $\psi$ and with the topology as defined above, is étale.

Proof. For any $\left(e_{1}, e_{2}\right)$ in $G_{E^{\psi}}$ and any open neighbourhood $U$ of $r\left(e_{1}\right)=r\left(e_{2}\right)$, the restriction of the head and tail maps on the neighbourhood $O\left(\left(e_{1}, e_{2}\right), U\right)$ of $\left(e_{1}, e_{2}\right)$ are homeomorphisms since, for example, for $\left(e_{1}^{1}, e_{2}^{1}\right)$ and $\left(e_{1}^{2}, e_{2}^{2}\right)$ in $O\left(\left(e_{1}, e_{2}\right), U\right)$ with $h\left(e_{1}^{1}, e_{2}^{1}\right)$ equal to $h\left(e_{1}^{2}, e_{2}^{2}\right)$, we have $e_{1}^{1}=e_{1}^{2}$ and $e_{2}^{1}=\left(\psi_{i}\left(r\left(e_{1}^{1}\right)\right), r\left(e_{1}^{1}\right)\right)=$ $\left(\psi_{i}\left(r\left(e_{1}^{2}\right)\right), r\left(e_{1}^{2}\right)\right)=e_{2}^{2}\left(\right.$ if $\left.s\left(e_{2}\right)=\psi_{i}\left(r\left(e_{2}\right)\right)\right)$.

Proposition 6.2. The groupoid algebra $C^{*}\left(G_{E^{\psi}}\right)$ is isomorphic to $M_{k}(C(Y))$.

Proof. For $y$ in $Y$, we consider the arrows $a_{i j}(y)$ with head equal to $\left(\psi_{i}(y), y\right)$ and tail equal to $\left(\psi_{j}(y), y\right)$ for $1 \leq i, j \leq k$. For any $f$ in $C^{*}\left(G_{E^{\psi}}\right)$, we define

$$
\tilde{f}(y):=\left(f\left(a_{i j}(y)\right) \in M_{k}(\mathbb{C})\right.
$$

for all $y$ in $Y$.

We claim that $f \mapsto \tilde{f}$ is a ${ }^{*}$-isomorphism from $C^{*}\left(G_{E^{\psi}}\right)$ to $M_{k}(\mathbb{C})$. For fixed $i, j$, the map $f\left(a_{i j}(\cdot)\right)$ from $Y$ to $\mathbb{C}$ is continuous by the definition of the topology on $G_{E^{\psi}}$. So the map $f \mapsto \tilde{f}$ maps $C^{*}\left(G_{E^{\psi}}\right)$ to $M_{k}(C(Y))$. Moreover, for $y$ in $Y$, the $(i, j)$-th entry of the image is

$$
\begin{aligned}
(\widetilde{f * g})_{i j}(y) & =(f * g)\left(a_{i j}(y)\right) \\
& =\sum_{l=1}^{k} f\left(a_{i l}(y)\right) g\left(a_{l j}(y)\right) \\
& =\sum_{l=1}^{k} \tilde{f_{i l}}(y) \tilde{g_{l j}}(y) .
\end{aligned}
$$


This implies $(\widetilde{f * g})(y)=\tilde{f}(y) \tilde{g}(y)$. It is clear from the definition that $\widetilde{f *}(y)=$ $(\tilde{f})^{*}(y)$, and hence the map is a ${ }^{*}$-homomorphism. Injectivity and surjectivity also follow from the definition of the map. This completes the proof of the assertion.

We can define another groupoid which is a subgroupoid of $G_{E^{\psi}}$, namely,

$$
G_{E^{\psi}}^{0}:=\left\{\left(e_{1}, e_{2}\right) \in G_{E^{\psi}}: s\left(e_{1}\right)=s\left(e_{2}\right)\right\}
$$

endowed with the induced topology.

Proposition 6.3. The commutative $\mathrm{C}^{*}$-algebra $C(\operatorname{Image}(\psi))$ is a sub- $\mathrm{C}^{*}$-algebra of $C^{*}\left(G_{E \psi}^{0}\right)$.

Proof. Let $f$ be an element in $C(\operatorname{Image}(\psi))$. We define $\hat{f}$ on $G_{E \psi}$ by

$$
\hat{f}\left(\left(e_{1}, e_{2}\right)\right):=f\left(s\left(e_{1}\right)\right)\left(=f\left(s\left(e_{2}\right)\right)\right) .
$$

Then the map $f \mapsto \hat{f}$ is an injective *-homomorphism from $C(\operatorname{Image}(\psi))$ to $C^{*}\left(G_{E \psi}^{0}\right)$.

If we define

$$
\iota(f)\left(\left(e_{1}, e_{2}\right)\right)=\left\{\begin{array}{lr}
f\left(e_{1}, e_{2}\right) & e_{1}=e_{2}, \\
0 & \text { otherwise },
\end{array}\right.
$$

for $f$ in $C^{*}\left(G_{E^{\psi}}^{0}\right)$ and $\left(e_{1}, e_{2}\right)$ in $G_{E^{\psi}}$, then $\iota$ is a canonical imbedding of $C^{*}\left(G_{E^{\psi}}^{0}\right)$ into $C^{*}\left(G_{E^{\psi}}\right)$.

Now, we can give a partial answer to question $(Q 1)$.

Theorem 6.1. For any non-singular abstract eigenvalue pattern $\psi: Y \rightarrow P^{k}(X)$ with the global labelling property, there exists a ${ }^{*}$-homomorphism $\psi^{*}: C(X) \rightarrow$ $M_{k}(C(Y))$ having $\psi$ as its eigenvalue pattern.

Proof. For a function $f$ in $C(X)$, by Proposition 6.3 we can regard the restriction of $f$ to the image of $\psi$ as an element of the groupoid algebra $C^{*}\left(G_{E^{\psi}}\right)$. Now the map $\psi^{*}: f \mapsto \iota\left(\left.f\right|_{\text {image }(\psi)}\right)$ is a ${ }^{*}$-homomorphism from $C(X)$ to $C^{*}\left(G_{E^{\psi}}\right)$, which is isomorphic to $M_{k}(C(Y))$ by Proposition 6.2. This map has $\psi$ as its eigenvalue pattern, as desired.

\section{LOCAL LABELLING PROPERTY AND NON-SINGULAR EIGENVALUE PATTERNS ON THE CIRCLE}

In the case $X=Y=\mathbb{T}$, the one-dimensional circle, there are many abstract eigenvalue patterns which do not satisfy the global labelling property. One simple example is the following one:

If we parametrize $\mathbb{T}$ by the set $\left\{e^{2 \pi i t}: t \in(0,1]\right\}$ in $\mathbb{C}$, then the eigenvalue pattern $\phi: \mathbb{T} \rightarrow P^{2}(\mathbb{T}), e^{2 \pi i t} \mapsto\left[\left(e^{\pi i t}, e^{\pi i t+\pi i}\right)\right]$, has no global labelling. Geometrically, the graph of $\phi$ is:

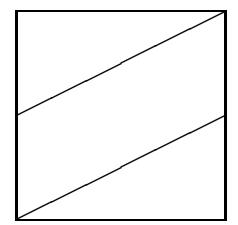

We see that, at $t=1$, the pattern flips over. Fortunately, a suitable topology can be introduced on the groupoid $G_{E^{\phi}}$ to make it an étale groupoid. 
More generally, let there be a given non-singular abstract eigenvalue pattern $\psi: Y \rightarrow P^{k}(X)$, where $X$ and $Y$ are compact Hausdorff spaces. The eigenvalue pattern $\psi$ satisfies the Local Labelling Property if for any $y$ in $Y$, there exist an open neighbourhood $U$ of $y$ and continuous functions $\psi_{1}, \ldots, \psi_{k}$ from $U$ to $X$ with $\psi\left(y^{\prime}\right)=\left\{\psi_{1}\left(y^{\prime}\right), \ldots, \psi_{k}\left(y^{\prime}\right)\right\}$ (up to permutation) for any $y^{\prime}$ in $U$.

Suppose that the eigenvalue pattern $\psi: Y \rightarrow P^{k}(X)$ satisfies the local labelling property. We can fix a local labelling of $\psi$ : by the compactness of $Y$, we can choose an open covering $\left\{v_{i}\right\}_{j=1}^{l}$ of $Y$ with labelling $\left(\psi_{1}^{i}, \ldots, \psi_{k}^{i}\right)$ on each $v_{i}, i=1, \ldots, l$.

Let $\left(e_{1}, e_{2}\right)$ be an element in the groupoid $G_{E \psi}$ and let $U$ be an open neighbourhood of the element $r\left(e_{1}\right)\left(=r\left(e_{2}\right)\right)$ in $Y$. Since the sets $\left\{v_{i}\right\}_{i=1}^{l}$ cover $Y$, the element $r\left(e_{1}\right)\left(=r\left(e_{2}\right)\right)$ is in the intersection of $U$ and $v_{i}$ for some $i$. By the local labelling property, the source element $s\left(e_{1}\right)$ equals $\psi_{j_{1}}^{i}\left(r\left(e_{1}\right)\right)$, and the other source element $s\left(e_{2}\right)$ equals $\psi_{j_{2}}^{i}\left(r\left(e_{2}\right)\right)$ for some $1 \leq j_{1}, j_{2} \leq l$. We define

$O\left(\left(e_{1}, e_{2}\right), U\right):=\left\{\left(e, e^{\prime}\right): r(e)=r\left(e^{\prime}\right) \in U, s(e) \in\left(\psi_{j 1}^{i}\right)^{-1}(U), s\left(e^{\prime}\right) \in\left(\psi_{j 2}^{i}\right)^{-1}(U)\right\}$.

Topology on the groupoid $G_{E^{\psi}}$ is generated by such sets.

Remarks.

(1) Endowed with this topology, the groupoid $G_{E^{\psi}}$ is étale.

(2) This topology is not canonical: two different local labellings induce two different topologies.

(3) For the case $Y$ equal to the one-dimensional circle, there is a Möbulis-bandparadox: if we move an arrow in the groupoid $G_{E^{\psi}}$ around the circle once along the local labelling covering, we will not get the original arrow. It is just like the mobulis band being twisted (you can get the original arrow if you move around the circle twice). In our case, in order to get the original arrow, we need to move around the circle $k$ ! times, which is the order of the permutation group with $k$ elements.

We have the same result as in the global labelling case (Theorem 6.1). (In other words, the global hypothesis of Theorem 6.1 can be replaced by the local one.)

Theorem 7.1. For two compact Hausdorff spaces $X$ and $Y$, with $Y$ being one dimensional and any non-singular abstract eigenvalue pattern $\psi: Y \rightarrow P^{k}(X)$ satisfying the local labelling property, there exists a ${ }^{*}$-homomorphism $\psi^{*}: C(X) \rightarrow$ $M_{k}(C(Y))$ having $\psi$ as its eigenvalue pattern.

Proof. The argument of the statement in the global labelling property case can be carried over. One minor change in the proof is that the range of the map $f \mapsto \tilde{f}$ (Proposition 6.2) is a $\mathrm{C}^{*}$-bundle over $Y$ having fibre, at each $y$ in $Y$, isomorphic to $M_{k}(\mathbb{C})$, so the range is isomorphic to a sub- $C^{*}$-algebra of $M_{k}(C(Y))$.

Therefore, we have an affirmative answer to question $(Q 1)$ in the case $X=Y=$ $\mathbb{T}$, the one dimensional circle:

Corollary 7.1. Any $k$-dimensional non-singular abstract eigenvalue pattern on $\mathbb{T}$ is the eigenvalue pattern of some homomorphism from $C(\mathbb{T})$ to $M_{k}(C(\mathbb{T}))$

Proof. Any such eigenvalue pattern satisfies the local labelling property, since for each point in $\mathbb{T}$, we can choose a neighbourhood such that the image of that eigenvalue pattern is not surjective. Hence we can cut the circle $\mathbb{T}$ at a point to make the image to be on a closed interval. Then we can label that neighbourhood by the 
image ordering on that closed interval. Using the compactness of the circle $\mathbb{T}$, we get a local labelling.

\section{A Singular CASE}

In previous sections, we considered the maps with $C(X)$ as their domains. In the following two sections, we extend the definition of an eigenvalue pattern to a singular form which corresponds to a map with a sub-C*-algebra of $M_{k}(C(X))$ as its domain.

In particular, we consider a case with $X$ equal to the interval $[0,1]$ and having one singular point (at the fibre at infinity; see the definition below). We are interested in this special case because it has an application on classifying simple inductive limits of algebras having these properties as building blocks (cf. Ts1], Ts2]).

Clearly, the ideas in these two sections can be extended to other cases (for example, having different spectra or different number of singular points) without difficulty.

For a pair of natural numbers $\left(n, n^{\prime}\right)$ with $n$ dividing $n^{\prime}\left(n^{\prime}>n\right)$, let $M_{n}(\mathbb{C})$ and $M_{n^{\prime}}(\mathbb{C})$ denote the matrix algebras over $\mathbb{C}$ of orders $n$ and $n^{\prime}$, respectively. Let homomorphisms $\phi_{0}$ and $\phi_{1}$, from $M_{n}(\mathbb{C})$ to $M_{n^{\prime}}(\mathbb{C})$, have multiplicities $\frac{n^{\prime}}{n}-1$ and $\frac{n^{\prime}}{n}$, respectively. (Up to unitary equivalence, the homomorphism $\phi_{0}$ maps $M_{n}(\mathbb{C})$ into diagonal block matrices in $M_{n^{\prime}}(\mathbb{C})$ with $\frac{n^{\prime}}{n}-1$ identical blocks and one zero block, and the homomorphism $\phi_{1}$ yields matrices with $\frac{n^{\prime}}{n}$ identical blocks; in particular, the homomorphism $\phi_{1}$ is unital.) We define

$$
A\left(n, n^{\prime}\right):=\left\{f \in M_{n^{\prime}}(C[0,1]): f(0)=\phi_{0}(c), f(1)=\phi_{1}(c), c \in M_{n}(\mathbb{C})\right\} .
$$

The irreducible representations of $A\left(n, n^{\prime}\right)$ are just the natural evaluation maps. More precisely, for $0<s<1$, consider the representation $e_{s}: A\left(n, n^{\prime}\right) \rightarrow M_{n^{\prime}}(\mathbb{C})$ of $A\left(n, n^{\prime}\right)$ obtained by evaluating at $s$. Also consider the representation $e_{\infty}$ : $A\left(n, n^{\prime}\right) \rightarrow M_{n}(\mathbb{C})\left(e_{\infty}(f)=c\right.$ if $\left.f(0)=\phi_{0}(c)\right)$ of $A\left(n, n^{\prime}\right)$ obtained by evaluating at the irreducible fibre at infinity. Then every irreducible representation of $A$ is unitarily equivalent either to $e_{s}$ for some $s \in(0,1)$ or to $e_{\infty}$. In other words, the spectrum of $A\left(n, n^{\prime}\right)$ is $\mathbb{T}$, the one-dimensional circle.

If we parametrize the spectrum of $A\left(n, n^{\prime}\right), \mathbb{T}$, by $\left\{e^{2 \pi i t}: t \in(0,1]\right\}$ (with $e^{2 \pi i 1}=1$ representing the irreducible representation $e_{\infty}$ at the fibre at infinity), we see that the point $e^{2 \pi i 1}=1$ is a singular point since when $t$ tends to 1 , the

irreducible representation $e_{t}$ tends to the direct sum of $\frac{n^{\prime}}{n}$ copies of the irreducible representation $e_{\infty}$. On the other hand, when $t$ tends to 0 , the irreducible representation $e_{t}$ tends to the direct sum of $\frac{n^{\prime}}{n}-1$ copies of the irreducible representation $e_{\infty}$

Example. Let us consider the map

$$
\begin{aligned}
\Phi & : A(1,2) \rightarrow M_{4}(\mathbb{C}), \\
\Phi(f)(t)= & \left\{\begin{array}{cc}
\left(\begin{array}{cc}
f(2 t) & 0 \\
0 & 0
\end{array}\right), \\
u_{t}\left(\begin{array}{cc}
f(2 t-1) & 0 \\
0 & f(2 t-1)
\end{array}\right) u_{t}^{*}, & t \in\left(\frac{1}{2}, 1\right],
\end{array}\right.
\end{aligned}
$$


where $u_{t}$ is a continuous path of unitary elements in $M_{4}(\mathbb{C})$ connecting $u_{\frac{1}{2}}=$ $\left(\begin{array}{llll}1 & 0 & 0 & 0 \\ 0 & 0 & 1 & 0 \\ 0 & 1 & 0 & 0 \\ 0 & 0 & 0 & 1\end{array}\right)$ to $u_{1}=$ identity element of $M_{4}(\mathbb{C})$; this path makes the image continuous at $t=\frac{1}{2}$.

According to the definition in Section 2, the eigenvalue pattern of $\Phi$ is the map

$$
\begin{aligned}
\Phi^{*}:[0,1] & \rightarrow\left\{\begin{array}{lr}
1, & t=0, \\
\left\{\frac{1}{2} t\right\}, & t \in\left(0, \frac{1}{2}\right], \\
\{2 t-1,2 t-1\}, & t \in\left(\frac{1}{2}, 1\right), \\
\{1,1,1,1\}, & t=1,
\end{array}\right.
\end{aligned}
$$

or geometrically,

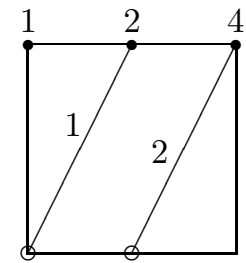

The numbers represent the multiplicities at different regions.

From this example, we can see that the total multiplicity of a singular eigenvalue pattern may not be fixed. More precisely, total multiplicity may change at the singular point.

Therefore, in order to define a singular abstract eigenvalue pattern, the continuity of an abstract pattern must be compatible with the multiplicity change at the singular point.

\section{Singular abstract Eigenvalue pattern AND its Groupoid Algebra}

For two natural numbers $k$ and $N$ (usually $k$ is greater than $N$ ), we denote the family of all finite subsets of $\mathbb{T}$ (with multiplicity) with at most $k$ elements as $\mathbb{T}_{N}^{k}$.

We endow the space $\mathbb{T}_{N}^{k}$ with the $N$-singular Gromov-Hausdorff topology (the topological structure at non-singular points is the same as the usual GromovHausdorff topology; at the singular point 1 , an element $\{x\}$ tends to $\{\underbrace{1,1, \ldots, 1,1}_{N-1 \text { times }}\}$ if $x$ tends to 0 ; on the other hand, an element $\{y\}$ tends to $\{\underbrace{1,1, \ldots, 1,1}_{N \text { times }}\}$ if $y$ tends to 1$)$.

A $k$-dimensional $N$-singular abstract eigenvalue pattern on $\mathbb{T}$ is a continuous map $\Psi$ from the interval $[0,1]$ to the space $\mathbb{T}_{N}^{k}$ with $k$ being the smallest integer such that the space $\mathbb{T}_{N}^{k}$ contains the image of $\Psi$.

For any $y$ in the interval $[0,1]$, let us write the image $\Psi(y)$ as $\left\{x_{1}^{y}, \ldots, x_{l_{y}}^{y}, 1, \ldots, 1\right\}$ with $x_{1}^{y}, \ldots, x_{l_{y}}^{y}$ non-singular. Each non-singular $x_{t}^{y}, 1 \leq t \leq l_{y}$, corresponds to an $N$-dimensional representation; therefore, we consider $N$ copies of $x_{t}^{y}$ (we index these copies by 1 to $N$ ) and the $N \times N$ arrows formed by these $N$ copies of $x_{t}^{y}$ (the arrows $\left(\left(y, x_{t i}^{y}\right),\left(y, x_{t j}^{y}\right)\right)$ with the $i$-th copy of $x_{t}^{y}$ as the source of its head and the $j$-th copy of $x_{t}^{y}$ as the source of its tail, for $\left.1 \leq i, j \leq N\right)$. On the other hand, we consider a single arrow to represent all the singular points. 
Now, let $G_{\Psi}$ be the collection of all such arrows with multiplicity ranging over $Y$. This collection of arrows, $G_{\Psi}$, has a structure of a groupoid:

(1) Two non-singular arrows $\left(e_{1}, e_{1}^{\prime}\right),\left(e_{2}, e_{2}^{\prime}\right)$ are composable if the head $e_{1}^{\prime}$ of the first arrow equals the tail $e_{2}$ of the second arrow (the indexes of the sources are the same).

(2) A singular arrow is composable only with itself and the composition is itself.

(3) The inverse of a non-singular arrow $\left(e, e^{\prime}\right)$ is $\left(e^{\prime}, e\right)$.

(4) The inverse of a singular arrow is itself.

Also, the topology on the groupoid $G_{\Psi}$ is defined as the following:

(1) If non-singular $x^{y}$ (in $\left.\Psi(y)\right)$ tends to 0 as $y$ tends to some point $y_{1}$ in $Y$, then the $N-1$ arrows, with the $i$-th copy of $x^{y}$ as its head and tail, $1 \leq i \leq N-1$, tends to the singular arrow at $y_{1}$. On the other hand, if non-singular $x^{y}$ (in $\Psi(y)$ ) tends to 1 as $y$ tends to some points $y_{2}$ in $Y$, then the $N$ arrows, with the $i$-th copy of $x^{y}$ as its head and tail, $1 \leq i \leq N$, tends to the singular arrows at $y_{2}$.

(2) Basic neighbourhoods of the non-singular arrows are defined as in Section 7 (with the indexes as labellings).

We note that the groupoid $G_{\Psi}$ may not be étale with this topology, but we can still define the groupoid algebra $C^{*}\left(G_{\Psi}\right)$, and by the same argument as in the proof of Theorem [7.1] we have $C^{*}\left(G_{\Psi}\right) \subseteq M_{k N}(C[0,1])$.

Furthermore, we consider the sub-C*-algebra $C^{*}\left(G_{\Psi}\right)_{0}$ of the groupoid algebra $C^{*}\left(G_{\Psi}\right)$, which is equal to $\left\{f \in C^{*}\left(G_{\Psi}\right): f\left(\left(e_{1}, e_{1}^{\prime}\right)\right)=f\left(\left(e_{2}, e_{2}^{\prime}\right)\right)\right.$ if $s\left(e_{1}\right) \equiv s\left(e_{2}\right)$ and $\left.s\left(e_{1}^{\prime}\right) \equiv s\left(e_{2}^{\prime}\right)\right\}$, where $s\left(e_{1}\right) \equiv s\left(e_{2}\right)$ means $s\left(e_{1}\right)$ is equal to $s\left(e_{2}\right)$ as an element in $\mathbb{T}$ and also with the same index. The inclusion map of this sub-C ${ }^{*}$-algebra into $C^{*}\left(G_{\Psi}\right)$ induces a ${ }^{*}$-homomorphism from $A(1, N)$ to $M_{k N}(C[0,1])$.

Finally, we have an analogous result for the singular case.

Theorem 9.1. Any $k$-dimensional $N$-singular abstract eigenvalue pattern on $\mathbb{T}$, the one-dimensional circle, is the eigenvalue pattern of some *-homomorphism from $A(1, N)$ to $M_{k N}(C[0,1])$.

\section{REFERENCES}

[Br] O. Bratteli, Inductive limits of finite dimensional $\mathrm{C}^{*}$-algebras, Trans. Amer. Math. Soc. 171 (1972), 195-234. MR0312282 (47:844)

[EHS] E. Effros, D. E. Handelman and C. L. Shen, Dimension groups and their affine representations, Amer. J. Math. 102 (1980), 385-407. MR0564479 (83g:46061)

[Ell1] G. A. Elliott, On the classification of $\mathrm{C}^{*}$-algebras of real rank zero, J. Reine Angew. Math. 443 (1993), 179-219. MR 1241132 (94i:46074)

[El12] G. A. Elliott, An invariant for simple C*-algebras. Invited Papers/Articles sollicités, Canadian Mathematical Society 1945-1995, Volume 3 (eds. J. B. Carrell and R. Murty), Canadian Mathematical Society, Ottawa, 1996, 61-90. MR1661611(2000b:46095)

[EV] G. A. Elliott and J. Villadsen, Perforated Ko-groups. Canad. J. Math. 52 (2000), 11641191. MR1794301 (2002j:46088)

[Eva] D. Evans, Quasiproduct states on $\mathrm{C}^{*}$-algebras. Operator algebras and their connections with topology and ergodic theory (Buşteni, 1983), 129-151, Lecture Notes in Math., 1132, Springer-Verlag, Berlin, 1985. MR0799567(87e:46079)

[Go] G. Gong, On the Classification of Simple Inductive Limit C*-Algebras, I: The Reduction Theorem, Documenta Math. 7 (2002), 255-461 MR2014489

[Ra] S. Razak, On the classification of simple stably projectionles $\mathrm{C}^{*}$-algebras, Canad. J. Math. 54 (2002), 138-224. MR1880962 (2003h:46091) 
[Re] J. Renault, A groupoid approach to $\mathrm{C}^{*}$-algebras, Lecture Notes in Mathematics 793, Springer-Verlag, Berlin, 1980. MR0584266 (82h:46075)

[Ts1] K. W. Tsang, A classification of certian simple stably projectionless $\mathrm{C}^{*}$-algebras, submitted.

[Ts2] K. W. Tsang, On the positive tracial cones of simple stably projectionless $\mathrm{C}^{*}$-algebras, to appear in J. Funct. Anal..

Department of Mathematics, University of Toronto, 100 St. George Street, Toronto, Ontario, Canada M5S 3G3

E-mail address: tsang@math.toronto.edu

Current address: Department of Mathematics, D3-2/F-09, The Hong Kong Institute of Education, 10 Lo Ping Road, Tai Po, Hong Kong

E-mail address: kwtsang@ied.edu.hk 